\title{
La mediación y la investigación para la paz: la búsqueda de alternativas pacíficas a los conflictos en la arena internacional ${ }^{*}$
}

\author{
Fernando HARTO DE VERA \\ Universidad Complutense de Madrid \\ fernandoharto@cps.ucm.es
}

Recibido: 04/06/2012

Aceptado: 13/11/2012

\begin{abstract}
Resumen
Una de las aplicaciones de la investigación para la paz ha sido generar técnicas para la resolución pacífica de los conflictos. El objetivo de este artículo es realizar una revisión del estado de la cuestión que dé cuenta de una de estas técnicas de resolución pacífica de los conflictos (la mediación) aplicada a una de las posibles dimensiones del conflicto (el conflicto internacional).

Palabras clave: investigación para la paz, resolución de conflictos, mediación
\end{abstract}

\section{Mediation and Peace Research: the search for peaceful alternatives to con- flicts in the international arena}

\begin{abstract}
One of the applications of peace research has been to generate techniques for peaceful resolutions of conflicts. This article review the state of art to account one of these techniques of peaceful resolution of conflicts (mediation) applied to one of the possible dimensions of conflict (the international conflict).
\end{abstract}

Keywords: peace research, conflict resolution, mediation

\section{Referencia normalizada}

Harto de Vera, F. (2013). "La mediación y la investigación para la paz: la búsqueda de alternativas pacíficas a los conflictos en la arena internacional”. Política y Sociedad, Vol.50 núm. 1: páginas. 5370

Sumario: Introducción. 1.Las técnicas de resolución pacífica de los conflictos. 2.Conclusiones. 3.Referencias bibliográficas

* Agradezco los comentarios y observaciones de los evaluadores anónimos que han sido tenidos en cuenta en la redacción final de este trabajo. 


\section{Introducción}

La Investigación para la paz nace tras el final de la Segunda Guerra Mundial en los Estados Unidos. Su objetivo fundacional fue configurarse como una disciplina científica unificada por una teoría general del conflicto, de carácter universal, integrada e interdisciplinar. Esta ambiciosa pretensión fue abandonada a finales de los años sesenta del siglo XX. A partir de ese momento se reconoció la imposibilidad de lograr dicho objetivo. En su lugar, el área experimentó un proceso doble. Por un lado, se asistió a su fragmentación y a la especialización en función de los distintos niveles del conflicto (internacional, interpersonal, intrapersonal). Por otro, el esfuerzo de investigación se dirigió hacia el descubrimiento, aplicación y extensión de técnicas prácticas para la resolución de conflictos en todos los niveles del mismo y en muy variados contextos de la realidad. Esta es la situación que prevalece en nuestros días ${ }^{1}$.

Y es que desde los inicios de la investigación para la paz ha habido una pregunta que ha ocupado la atención de los especialistas: ¿Para que sirve la investigación para la paz?. En el debate que ha generado este interrogante se han dado diferentes respuestas. Por lo que se refiere a la utilidad de la investigación para la paz, de acuerdo con Fisas (1987: 21-22) pueden distinguirse cuatro formas de aplicación:

1) Investigación.

a) Para fomentar la investigación de ciencias sociales o aplicadas dirigidas al desarrollo de bienes de utilidad social, señalando las contradicciones de determinadas orientaciones de la investigación.

b) Desarrollando y perfeccionando el análisis de las causas, estructuras y dinámicas de situaciones de paz, guerra y conflictos.

2) Mediación.

a) Proporcionando elementos conceptuales y metodológicos de resolución de conflictos.

3) Comportamiento político.

a) Facilitando juicios éticos y empíricos sobre las consecuencias de determinadas políticas diplomáticas, económicas, militares, sociales y culturales en relación a situaciones conflictivas.

4) Educación para la paz.

a) Proporcionando elementos de análisis, juicios y alternativas que permitan formar un comportamiento colectivo favorable a un mundo en paz.

${ }^{1}$ Un recorrido histórico sobre los principales hitos de la Investigación para la paz desde sus inicios hasta la actualidad puede consultarse en Harto (2005). 
Así pues, dado que una de las aplicaciones de la investigación para la paz ha sido la de generar técnicas para la resolución pacífica de los conflictos, la tarea que nos proponemos en este artículo es realizar una revisión del estado de la cuestión que de cuenta de una de estas técnicas de resolución pacífica de los conflictos (la mediación) aplicada a una de las posibles dimensiones del conflicto (el conflicto internacional).

\section{Las técnicas de resolución pacífica de los conflictos}

Tanto la paz como el conflicto pueden ser considerados normales en el sentido de que ninguna de las dos situaciones son anomalías o patologías. Ambos hechos se producen con la suficiente periodicidad como para que formen parte de la dinámica de las sociedades. Sin embargo, es indudable que una relación de tipo conflictivo acarrea una serie de costes. Costes de tipo económico, puesto que el conflicto requiere una acumulación de recursos para tratar de lograr una posición ventajosa sobre el adversario. Pero, además, existen una serie de costes para los participantes o actores enfrentados entre sí. No hay duda de que las situaciones de conflicto, una vez que se superan determinados niveles en la escalada del mismo, tienden a producir en los participantes una sensación de agotamiento debido a las energías empleadas. Esto es bien visible cuando la situación conflictiva es de tal magnitud que entraña el empleo de la violencia física. En estos casos, las energías y el esfuerzo requeridos para sostener el enfrentamiento con el enemigo son percibidos como un desgaste excesivo que en algún momento debe finalizar. El imaginario social de las colectividades que se han visto envueltas en situaciones de conflicto agudo revela que el hecho de los combates marca un hito en la percepción que el grupo tiene de su propia historia. Así, hay un antes y un después de la guerra. Un antes y un después definido por los límites espacio- temporales del conflicto en su fase crítica, convirtiéndose éste en punto de referencia que permanece con toda nitidez en la memoria colectiva.

En definitiva, lo que queremos poner de manifiesto es que todo conflicto tiene un comienzo y un final. El final del conflicto no tiene porqué significar que las causas que lo motivaron hayan perdido vigencia. El conflicto no es más que una fórmula entre otras posibles, para tratar de solucionar las diferencias que existen entre dos o más adversarios. Otra fórmula consiste en intentar llegar a un acuerdo a través de métodos que no entrañen violencia física. Ambos métodos poseen sus costes. Comenzamos señalando que el conflicto es costoso, pero el consenso también lo es. El pacto supone la dejación de derechos. Para nuestros propósitos analíticos, poco importa que aquellos sean legítimos o no. Lo relevante es que cada una de las partes enfrentadas siente que sus motivos están plenamente justificados, hasta el punto de ser capaces de entrar en liza con quien se atreva a dudar de dicha legitimidad. El acuerdo con quien hasta un momento dado ha sido considerado enemigo 
no es fácil; ésta es, por el contrario, una circunstancia que conlleva un precio a tener en cuenta.

Si las dos situaciones - conflicto y acuerdo - entrañan costes, el que las diferencias se soluciones a través de mecanismos pacíficos o mediante la violencia, depende del balance entre los costes de ambas. Así, cuando al menos uno de los contendientes evalúa que el coste del conflicto es demasiado elevado, en comparación con el coste de una solución negociada, se produce la detención del conflicto. Esto no significa que las causas que desencadenaron las hostilidades desaparezcan. Simplemente, la capacidad de uno o de ambos contendientes para dirimir sus diferencias a través de la violencia ha disminuido hasta un nivel tal que la solución consensuada aparece como una posibilidad atractiva:

"Los planes de paz han tenido todos éxito en eliminar algún motivo o tipo de conflicto, pero nunca la guerra misma [...] Porque los planes de paz, lo mismo que el pacifismo tradicional, no recurren sino a los aspectos históricos y episódicos de las guerras, que son su infraestructura permanente” (Bouthoul 1970: 51-52)

"Puede ocurrir que las partes en conflicto quieran cesar el combate solamente cuando reconozcan que sus objetivos no pueden ser alcanzados sino a un precio que no están dispuestas a pagar o, más generalmente, cuando lleguen a la conclusión de que la continuación del conflicto es menos atractiva que la paz. En ninguno de los casos quieren aceptar la derrota, aunque acepten detenerse sin haber logrado la victoria. En tales situaciones pueden sentirse impulsadas a explorar las posibilidades de un compromiso" (Coser 1970: 50)

Para Brunk (2000: 28-32), tal y como se refleja en el cuadro I, hay que distinguir tres grandes enfoques a la hora de poner en marcha estrategias de resolución pacífica de los conflictos: Resolución del conflicto (Conflict Resolution), Gestión del conflicto (Conflict Management) y Transformación del conflicto (Conflict Transformation). Cada uno de estos tres enfoques se caracteriza por sostener una concepción de la paz diferente, como se muestra en la primera fila del cuadro I. A su vez, dentro de cada uno de estos tres enfoques es posible el empleo de métodos coercitivos (violentos) y no coercitivos (no violentos), como se señala en las columnas. Finalmente, la última fila muestra algunos ejemplos de técnicas de resolución de los conflictos empleadas por estos tres enfoques.

En el primero de los enfoques, la "Resolución del conflicto", el objetivo fundamental consiste en la finalización del conflicto entre las partes de forma conjunta. La resolución del conflicto se muestra más apropiada y constructiva en aquellas situaciones en las que las partes aun percibiendo sus intereses como incompatibles sin embargo existe una solución que les permite a ambas la consecución de sus objetivos. Técnicas como la solución de problemas a través de la negociación, a menudo ayudada por la presencia de un mediador, son las más empleadas en este enfoque.

El segundo de los enfoques, la "Gestión del conflicto", tiene como objetivo principal más que el fin del conflicto, conseguir que las partes convivan con la relación conflictiva de tal manera que la mantengan dentro de unos límites que resulten beneficiosos para ambas. La metáfora que ejemplifica este tipo de enfoque la cons- 
tituyen los deportes: un partido de hockey o una partida de ajedrez es una situación conflictiva en la que dos contendientes se sujetan a unas reglas previamente aceptadas por ambos. En la vida real muchos conflictos se asemejan a juegos competitivos. Por ejemplo, la mayoría de los conflictos políticos dentro de las sociedades nunca pueden ser totalmente terminados. Precisamente una de las funciones de un sistema político que funcione correctamente consiste en encontrar normas, reglas y mecanismos que puedan ser aceptadas por colectividades plurales sin acabar con la diversidad y el pluralismo. La democracia, considerada desde este punto de vista, no es más que un sistema de gestión e institucionalización de los conflictos a través de mecanismos como el voto (la regla de la mayoría), el establecimiento de normas para asegurar el respeto a las minorías y la creación de tribunales de justicia autorregulados que tienen capacidad para imponer acuerdos de obligado cumplimiento a las partes (lo que, por otra parte, no significa el fin del conflicto entre ellas, sino solamente su gestión de modo pacífico). Uno de los mecanismos más usados en este enfoque es el "compromiso". En un compromiso, las partes en disputa acuerdan no el fin del conflicto sino la voluntad de convivir con él de modo que les permita a ambas conseguir los objetivos que pretenden. Las relaciones entre patronal y sindicatos, son un buen ejemplo de este tipo de situación.

Finalmente, el tercero de los enfoques la "Transformación del conflicto" consiste en perseguir la finalización del conflicto con la consecución de una paz justa, esto es, que elimine tanto el conflicto como las causas que lo provocaron. Es un enfoque

Cuadro 1: Tipos de resolución de conflictos

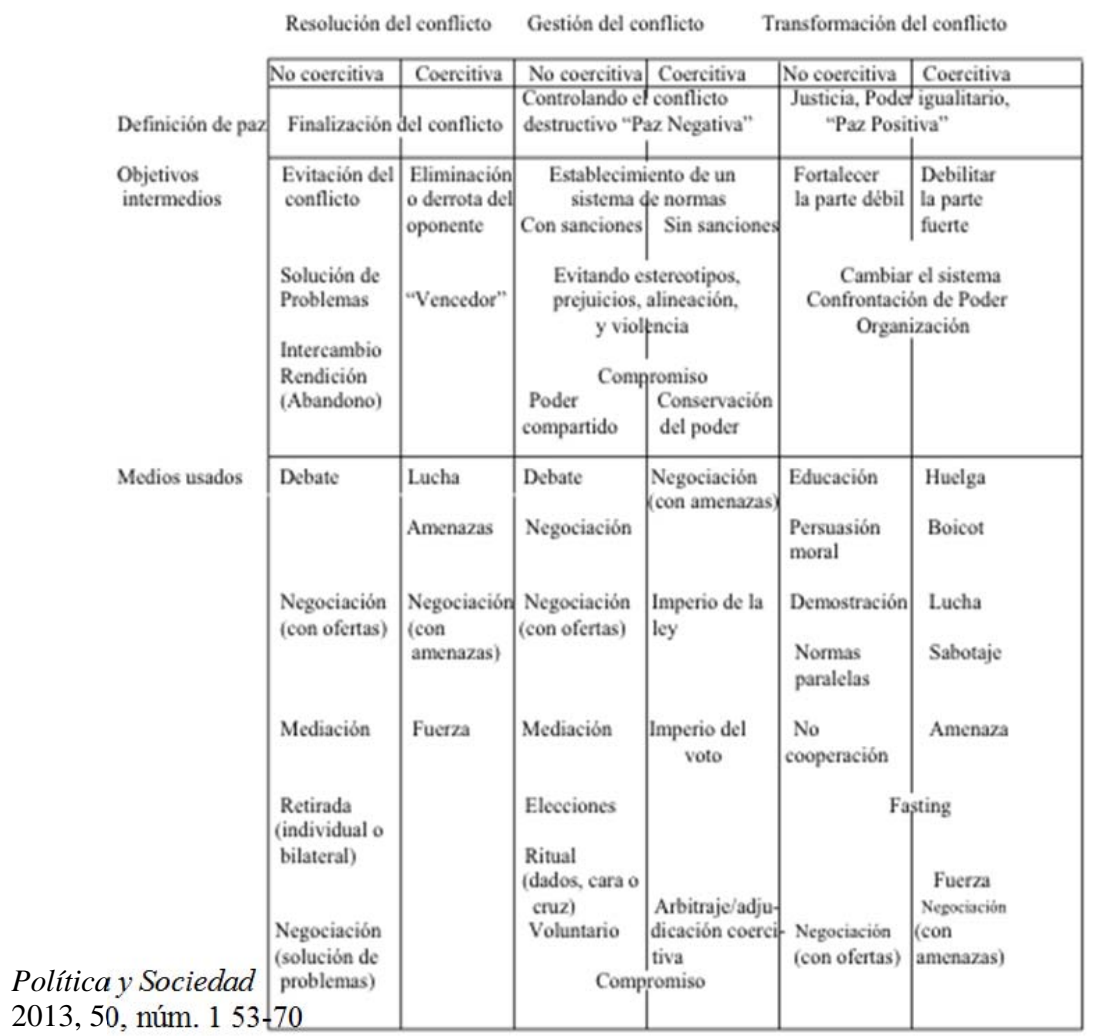


no exento de polémica, por cuanto plantea que frente al establecimiento de una paz negativa, es preferible la intensificación de los niveles de conducta conflictiva con el objetivo de lograr la paz positiva, única situación realmente pacífica y estable. Este enfoque sostiene que antes de poder resolver un conflicto es necesario atender al balance de poder entre las partes. Si este balance es manifiestamente desequilibrado entonces no es deseable entablar un proceso de negociación, mediación, solución de problemas o cualquier otra técnica de solución pacífica de las controversias puesto que el resultado del mismo será claramente favorable a los intereses de la parte que tenga más poder, produciendo de este modo un acuerdo injusto. En estos casos es necesario ensayar técnicas para lograr una mayor igualdad en la distribución del poder entre ambos contendientes. Otra situación que puede producirse es cuando una de las partes gana mucho más con el conflicto que la otra. En este tipo de situaciones la parte que se beneficia más con el conflicto puede no tener demasiado interés en reconocer que existe un conflicto que hay que solucionar e incluso que aún en el caso de que admita la existencia del mismo no tenga ninguna motivación para ensayar su resolución. Cuando se producen ambas situaciones el primer paso en el proceso de resolución del conflicto consiste en que la parte más débil desarrolle estrategias para captar la atención y ser tenida en cuenta por la parte más poderosa. Por eso el enfoque de la Transformación del conflicto es confrontacional por naturaleza. Su objetivo intermedio es encontrar mecanismos para motivar hacia la paz a la parte que carece de esta motivación. Uno de estos mecanismos consiste en el fortalecimiento de la parte más débil o en el debilitamiento de la parte más poderosa. Técnicas de presión, violentas y no violentas, tales como huelgas, boicots o desobediencia civil son propugnadas por este enfoque, para la consecución de este objetivo intermedio como paso previo al logro del objetivo último, esto es, la paz positiva.

La solución negociada de un conflicto, es decir, de modo que no implique la victoria de alguna de las partes, se produce como fruto de la interacción de ambos contendientes. Como mínimo, hay dos situaciones potenciales que pueden favorecer el inicio de las negociaciones. En primer lugar, la parte potencialmente vencida, aquella que experimenta la peor suerte, puede solicitar al adversario el fin de las hostilidades, al percibir que el coste de seguir manteniendo el conflicto es superior a la alternativa de la negociación. La segunda situación se produce cuando ambas partes advierten que ninguna de las dos tiene posibilidades de vencer al adversario. En ese momento la guerra pierde su carácter instrumental, y se convierte en un juego perverso de destrucción por la destrucción, incapaz de producir réditos en el sentido de asegurar la obtención de los objetivos que desataron el conflicto.

De acuerdo con Bercovitch (1997:125) hay cuatro estrategias de gestión de los conflictos: 1) violencia e imposición 2) disuasión 3) procedimientos judiciales y 4) estrategias de conciliación (negociación y mediación). Una vez que alguna de las dos situaciones a las que se refería el párrafo anterior se ha producido, se descartan las dos primeras estrategias, abriéndose así el espacio para que los contendientes ensayen alguna de las técnicas de resolución pacífica de los conflictos. En este sentido, la carta de las Naciones Unidas reconoce la existencia de tres técnicas 
básicas para la gestión pacífica de los conflictos internacionales. Estas tres técnicas son: 1) negociación directa entre las partes contendientes 2) diversas formas de mediación, buenos oficios y conciliación 3) arbitraje y procedimientos judiciales en los que una tercera parte tiene el poder de decisión. Simplificando podemos reducir a tres los modos de resolución pacífica de los conflictos: negociación, mediación y arbitraje.

La negociación es un procedimiento de resolución del conflicto entre dos o más partes enfrentadas que consiste en el desarrollo de discusiones a través de representantes oficiales, durante el curso de las cuales una o todas las partes modifican sus demandas, con el fin de llegar a un acuerdo aceptable para todos.

La mediación es un tipo de negociación en el sentido anteriormente descrito, en la cual interviene una tercera parte neutral cuya función es facilitar el acuerdo entre las partes contendientes.

El arbitraje se produce cuando los adversarios recurren a una tercera parte neutral con la intención de someter las diferencias a su criterio para que solucione la controversia. La figura del árbitro se asemeja a la del juez, puesto que ambas partes coinciden en que la decisión del árbitro será inapelable. Así, mientras que el mediador tiene un papel puramente funcional, el árbitro goza de poder de decisión,

\subsection{La mediación}

De acuerdo con Bercovitch (1997: 132-133) en la bibliografía sobre mediación en el conflicto internacional es posible distinguir cuatro enfoques:

1) Estudios prescriptivos: este enfoque está constituido por autores que ofrecen consejos sobre lo que constituye una buena práctica de la gestión de los conflictos a través de la mediación en situaciones del mundo real. Un buen ejemplo lo constituye la obra de Fisher y Ury (1981), auténtico best- seller. Estos estudios, desarrollados fundamentalmente por el "Programa sobre Negociación” de la Universidad de Harvard, han generado textos sobre cual debe ser el comportamiento de negociadores y mediadores y como los conflictos pueden ser resueltos.

2) Estudios teóricos: Realizados por académicos y negociadores tienen como objetivo el dearrollo de una teoría general para la resolución de los conflictos a través de la contrastación de modelos e hipótesis. Estos estudios utilizan diversas técnicas de interacción y de resolución de problemas (problem solving) para combinar la investigación experimental con la acción política. Algunos de sus representantes son (Burton 1969,1972,1984; Doob 1971; Fisher 1983; Kellman 1992; Walton 1969).

3) Estudios basados en la Teoría de Juegos: Realizados por economistas y teóricos de los juegos desarrollan modelos matemáticos para examinar como los sujetos en condiciones de máxima racionalidad y conocimiento se comportarían ante situaciones de conflicto. Estos estudios ilustran acerca de las 
estrategias más eficientes a la hora de realizar concesiones y lograr acuerdos (Raiffa 1982).

4) Estudios de caso: Son descripciones y análisis de situaciones de mediación que se han producido. Estos estudios persiguen desarrollar teorías y ofrecer líneas generales de actuación mediante a) la descripción detallada de un caso particular de mediación internacional (Ott 1972; Rubin 1981) b) enfoques experimentales y de laboratorio (Bartunek et al. 1975; Rubin 1980) para descubrir como las partes y el mediador se comportan en situaciones controladas y c) estudios sistemáticos de largo alcance que se basan en numerosos casos de mediación internacional para formular y contrastar proposiciones sobre la mediación eficiente así como para establecer las condiciones bajo las que la mediación puede funcionar mejor (Bercovitch y Rubin 1992; Touval y Zartman 1985).

Durante muchos años el estudio de la mediación ha sufrido de imprecisión conceptual y de falta de información. Asimismo nadie creía en la posibilidad de poder extraer de los casos particulares pautas generales que pudieran ser de validez universal a cualquier proceso de mediación. El enfoque más útil en el estudio de la mediación es el que establece una relación con otra de las estrategias de gestión pacífica de los conflictos: la negociación. La base de este enfoque es establecida por Stevens y Schelling. Para Stevens (1963: 123) "la mediación, como otros fenómenos sociales, es susceptible de análisis sistemático. La clave de este análisis está en el reconocimiento de que allí donde la mediación es empleada forma parte integral del proceso de negociación[...] Un análisis de la mediación no es posible más que en el contexto general del análisis de la negociación”. Por su parte Schelling (1960: 22) señala que la mejor manera de concebir a un mediador "es probablemente como un elemento de los dispositivos de comunicación o como una tercera parte con un valor específico propio".

La mediación es, al menos estructuralmente, la continuación de las negociaciones por otras vías. ¿Cuáles son entonces los elementos distintivos y propios de la mediación?:

1) La mediación es una extensión y continuación de la gestión pacífica del conflicto.

2) La mediación comprende la intervención de un "outsider"-un individuo, un grupo o una organización- en un conflicto entre dos o más estados u otros actores.

3) La mediación es una intervención no coactiva, no violenta y no obligatoria.

4) Los mediadores intervienen en un conflicto para influir en él, cambiarlo, resolverlo o modificarlo.

5) Los mediadores llevan consigo, consciente o inconscientemente, ideas conocimientos, recursos e intereses, propios o del grupo u organización al que representan. Los mediadores a menudo tienen sus propias asunciones y agendas sobre el conflicto en cuestión. 
6) La mediación es una forma voluntaria de gestión del conflicto. Los actores implicados mantienen el control sobre el resultado de su conflicto así como la libertad de aceptar o rechazar la mediación y las propuestas del mediador.

7) La mediación opera solo sobre bases ad hoc.

La mediación se distingue de otras técnicas como la negociación y el arbitraje. Con respecto a la negociación se caracteriza por una estructura triangular frente a la estructura binominal propia de la negociación. Respecto al arbitraje se distingue por su carácter no obligatorio. Existen distintas definiciones de mediación que podemos agrupar en tres grandes líneas según se subraye a) las ventajas que la presencia del mediador puede introducir b) la distinción entre la mediación y otros procesos de intervención de terceras partes y c) la descripción de los atributos del mediador.

Una definición que se ajusta al primer grupo es la que ofrece Oran Young (1967: 34): "cualquier acción llevada a cabo por un actor que no es parte directa en la crisis y que se dirige a reducir o eliminar uno o más de los problemas en la relación negociadora así como a facilitar la terminación de la propia crisis”. En esta misma línea Mitchell (1981: 287) define la mediación como "cualquier actividad de intermediación realizada por una tercera parte con la intención de alcanzar un compromiso sobre los asuntos que disputan las parte 0 , al menos, de finalizar la conducta conflictiva”. Por último, Blake y Mouton (1985: 15) se refieren a la mediación como un proceso que comprende "la intervención de una tercera parte que primero investiga y define el problema y después generalmente se aproxima por separado a cada grupo con recomendaciones dirigidas a promover una solución mutuamente aceptable".

Ejemplo del segundo grupo de definiciones lo proporciona Moore (1986: 6) al considerar a la mediación como "una extensión y elaboración del proceso de negociación. La mediación comprende la intervención de una tercera parte neutral, imparcial y aceptada que no tiene poder de decisión sobre las partes contendientes para imponer un acuerdo mutuamente aceptable por éstas”. En esta misma línea se pronuncia Singer (1990: 20) cuando señala que la mediación es "una forma de asistencia de una tercera parte que involucra a un "outsider" en la disputa que no tiene poder para oligar a tomar decisiones a las partes". Otras definiciones subrayan la neutralidad e imparcialidad como las características distintivas de la mediación. Así, Bingham (1985: 5) define la mediación como "la asistencia de una tercera parte neutral en una negociación”. Folberg y Taylor (1984: 7) ven la mediación como "el proceso por el que los participantes junto con la asistencia de una persona o personas neutrales, sistemáticamente aislan los puntos en disputa para desarrollar opciones, considerar alternativas y llegar a un acuerdo consensuado que responda a sus necesidades".

En cuanto al tercer grupo de definiciones, en la literatura se ha prestado una atención considerable a la cuestión de los roles, funciones y comportamiento del mediador. Wall (1981) en una revisión exhaustiva de la literatura identifica más de un centenar de funciones y comportamientos. Jeffrey Rubin (1981) clasifica los roles del mediador al distinguir entre mediación formal (ej: secretario general de 
Naciones Unidas), mediación informal (ej: especialistas académicos), mediación individual (ej: Lord Owen), mediación realizada por un representante estatal (ej: por el Secretario de Estado norteamericano), mediación solicitada, mediación no solicitada, mediación consultiva, mediación directiva, mediación temporal, mediación permanente, mediación orientada a la resolución y mediación orientada a la relación. Cada uno de estos tipòs de mediadores tiene diferentes intereses, recursos y capacidades; igualmente el comportamiento de cada uno puede conducir a resultados diferentes. Por su parte Stulberg (1987) ofrece la siguiente lista de los roles del mediador: a) catalizador b) educador c) traductor d) creador de recursos e) portador de malas noticias f) agente de realidad y g) chivo expiatorio.

\subsection{Las estrategias del mediador}

En estrecha relación con los roles del mediador se encuentran sus estrategias de comportamiento. Kolb (1983) distingue dos tipos de estrategias: estrategias para el acuerdo (afectan a la esencia del conflicto) y estrategias de orquestación (gestionan la interacción). Por su parte, Stein (1985) se refiere a dos tipos de estrategias, las estrategias incrementales que segmentan un conflicto en asuntos menores y las estrategias comprensivas que tratan con todos los aspectos del conflicto. Carnevale (1986) sostiene que los mediadores pueden elegir entre cuatro estrategias fundamentales: integración (búsqueda de intereses comunes), presión (reducción del espectro de alternativas disponibles), compensación (intensificación del atractivo de determinadas alternativas) e inacción (dejar a las partes que interaccionen por si mismas).

Una de las principales funciones del mediador, contribuir a una comunicación más fluida entre las partes, se produce tanto en las reuniones plenarias o a tres bandas (mediador y partes enfrentadas) como en las reuniones por separado (mediador y una de las partes).

Por lo que se refiere a las reuniones plenarias, con frecuencia es el mediador el que preside la reunión. Desde esta posición de privilegio, es a él a quien corresponde convocar la reunión, determinar el procedimiento, suspender las reuniones o postergarlas, así como convocar reuniones de comisiones de trabajo para temas concretos. Ejerce el papel de moderador de la reunión, concediendo turnos de palabra, resumiendo la discusión o evitando los enfrentamientos personales, por ejemplo.

En cuanto a las reuniones por separado, con frecuencia su convocatoria queda a criterio del mediador. Generalmente esto se produce en las fases iniciales, en los primeros contactos, o bien cuando se presenta una posición que altera el cuadro hasta entonces existente, de tal forma que resulta conveniente que las delegaciones se reúnan por separado. De este modo se genera un clima más sereno, que asegura la reflexividad y receptividad de las delegaciones. También se presenta esta situación cuando se asiste a un estancamiento del proceso negociador. Si se produce la 
convocatoria de reuniones separadas, la función de limar los escollos que impiden una comunicación fluida entre ambas delegaciones es fundamental.

Una de las razones por las que el mediador es una figura eficiente para la función comunicativa, estriba en que las proposiciones y concesiones que transmite no son todavía oficiales, sino que tienen un carácter provisional y tentativo. De esta forma, cada parte conserva suficiente libertad para cuestionar lo que se ha ofrecido, puesto que no ha comprometido oficialmente su posición. La posibilidad de vuelta atrás, paradójicamente, da como resultado el progreso de la negociación, ya que no se encuentra sometida a trabas ni corsés.

Finalmente, el procedimiento de las reuniones por separado da al mediador una visión de conjunto del proceso de la que carecen las partes. Esta visión de conjunto opera como un mecanismo que sitúa al mediador en una posición de ventaja respecto a ambas delegaciones. La información privilegiada a su disposición le permite aconsejar a ambas partes sobre los espacios en los que se abre la posibilidad de acuerdo.

Pero de todas las tipologías que sobre las estrategias, roles y comportamientos del mediador que nos ofrece la bibliografía especializada, quizás la más extendida sea la propuesta por Touval y Zartman (1985). Los autores identifican tres categorías de comportamiento del mediador en un nivel ascendente de implicación. Esta tipología es particularmente útil porque se deduce de un modelo general de la mediación que incluye la información, la toma de decisiones y la influencia. Asimismo puede ser utilizada como herramienta para la investigación empírica a través de la aplicación de cuestionarios tras el proceso de mediación. La tipología se organiza a través de tres tipos de conducta del mediador, la comunicación, la formulación y la manipulación, como sigue:

1) Estrategias de Comunicación

- contactos con las partes

- obtención de la confianza y credibilidad de las partes

- organización de la interacción entre las partes

- identificación de los intereses y temas subyacentes

- clarificación de la situación

- prevención de aspectos colaterales

- desarrollo del entendimiento con las partes

- proporcionar información oculta

- transmisión de mensajes entre las partes

- estimulación de la comunicación relevante

- ofrecer evaluaciones positivas

- permitir la discusión de los intereses de todas las partes

2) Estrategias de Formulación

- elección del lugar de reunión

- control del ritmo de las reuniones

- control del ambiente físico 
- establecimiento del protocolo

- asegurar la privacidad de la mediación

- sugerir procedimientos

- destacar los intereses comunes

- reducir tensiones

- control del calendario

- tratar primero los asuntos simples

- estructurar la agenda

- ayudar al diseño de un esquema para un resultado aceptable

- ayudar a las partes a "salvar la cara"

- mantener el proceso centrado en los asuntos relevantes

- realizar sugerencias y propuestas sustantivas

- sugerir las concesiones que pueden conceder las partes

3) Estrategias de manipulación

- mantener a las partes en la mesa

- cambias las expectativas de las partes

- exigir responsabilidad con las concesiones

- informar a las partes del costo del desacuerdo

- proporcionar y filtrar información

- ayudar a los negociadores a deshacer un compromiso

- recompensar las concesiones hechas por las partes

- presionar a las partes para que muestren flexibilidad

- prometer recursos o amenazar con la retirada

- ofrecimiento para verificar los acuerdos

- manejar incentivos y penalizaciones

La tipología de Touval y Zartman permite analizar y comprender como se comportan los mediadores en su tarea. La elección de un tipo u otro de estrategia raramente es aleatoria. Por el contrario, es una decisión que se basa en factores que dependen tanto del tipo de conflicto que se trate como de factores internos del propio mediador. Así, por ejemplo, en conflictos de baja intensidad, las estrategias de comunicación pueden ser más efectivas mientras que en los conflictos de alta intensidad se requiere de un rol más activo del mediador y en estos casos las estrategias de manipulación se revelan como más útiles. En definitiva, para que las estrategias de mediación sean efectivas se requiere que sean congruentes tanto con la naturaleza del conflicto como con los intereses y objetivos del mediador.

\subsection{Modelos teóricos en los procesos de mediación.}

En la bibliografía especializada en las técnicas de resolución pacífica de conflictos un aspecto muy interesante es el dedicado a la formulación de modelos teóricos. Estos modelos son herramientas analíticas que tratan de dotar de coherencia y 
sistematicidad a las múltiples variables y dinámicas que se hayan presentes en los procesos de negociación y mediación. Pero mientras que en el caso de la negociación es posible encontrar una pluralidad de modelos teóricos, en la mediación la bibliografía es escasa a la hora de construcciones teóricas ${ }^{2}$. En este trabajo recogemos a continuación uno de estos modelos teóricos para los procesos de mediación.

\section{El modelo de McGrath.}

Desde presupuestos claramente lewinianos, McGrath (1966) formula un modelode negociación en el que introduce la figura del mediador. Distingue tres tipos de factores relevantes en la negociación con mediación: los factores que influyen sobre los negociadores, los factores debidos a la presencia del mediador y los factores derivados de la tarea y la situación.

\section{1) Los factores que influyen sobre los negociadores:}

La situación de negociación definida por McGrath consta de tres participantes, los dos negociadores delegados de las partes en conflicto y el mediador. El mediador no está presente en la negociación a título particular. Su presencia responde a una representación del sistema social más amplio, dentro del cual se encuadra el conflicto, $\mathrm{y}$ al cual pertenecen las partes enfrentadas.

Figura 1: El modelo de McGrath

\section{EL MODELO DE MCGRATH}

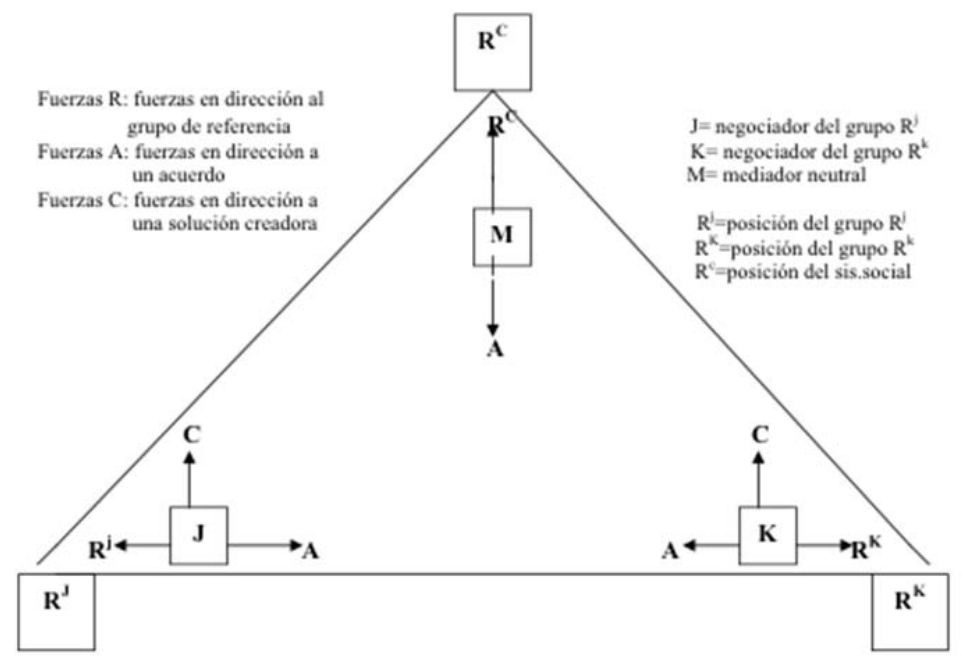

${ }^{2}$ Sin ánimo de ser exhaustivos es posible distinguir tres modelos teóricos para los procesos de negociación: el modelo de Sawyer y Guetzow, el modelo de Walton y Mc Kersie y el modelo de Stevens (Harto 2005). 
Los dos negociadores $(\mathrm{J}, \mathrm{K})$ y el mediador $(\mathrm{M})$ están sometidos a un sistema de fuerzas:

- Fuerzas R, que actúan en dirección al grupo que representa cada uno. En el caso de los negociadores, estas fuerzas los impulsan hacia la posición defendida por las respectivas organizaciones a las que representan. En el caso del mediador, el impulso se produce en dirección a la posición del sistema social al que representa ${ }^{3}$.

- Fuerzas A, que actúan en la dirección del acuerdo entre las partes.

- Fuerzas C, que impulsan hacia la consecución de una solución creativa y constructiva a ojos de un miembro del sistema social en el cual se inscribe el conflicto. Lógicamente, estas fuerzas sólo operan sobre las partes negociadoras, pero no sobre el mediador.

La resultante que impulsa la acción es el fruto de la combinación de las fuerzas R, $\mathrm{A}$, y C en el caso de los negociadores, y de la combinación de las fuerzas $\mathrm{R}$ y A en el caso del mediador.

2) Los factores debidos a la presencia del mediador:

El objetivo del negociador es doble. Por un lado, pretende el logro de una solución aceptable para ambas partes. Por otro, en su calidad de representante del público, le interesa cómo será recibido el resultado en el exterior. McGrath considera que el mediador es más bien la fuente de unas fuerzas que actúan sobre los negociadores y no el blanco de las fuerzas: es la fuente de una fuerza A y de una fuerza C. La intensidad de estas fuerzas afectará al proceso de negociación. A su vez, esta intensidad dependerá de factores tales como los conocimientos y la capacidad del mediador, la percepción del comportamiento del mediador por los negociadores y su relativa congruencia con las expectativas de dichos negociadores, y lo acertado de las tácticas que el mediador emplee en su misión.

3) Los factores que dependen de la tarea y la situación:

Estos factores influyen en el equilibrio de las fuerzas R, A y C que se ejercen sobre los negociadores. Están relacionados con los temas objeto de la negociación, la naturaleza de las partes enfrentadas y las reglas más o menos específicas de la negociación.

El último aspecto interesante del modelo de McGrath se refiere a los criterios para medir la eficacia de la negociación. Existen dos tipos de criterios de eficacia:

${ }^{3}$ En el interior de cualquier sistema social existe una pluralidad de intereses y posiciones. Pero esta pluralidad no impide que determinada posición sea la hegemónica, y en torno a ella se produzca el consenso que unifica y da organicidad al sistema. Es esta posición hegemónica la que orienta la fuerza $\mathrm{R}$ a la que se ve sometido el mediador. 
- Un criterio general objetivo, definido como el producto de la credibilidad de los tres jueces (las dos organizaciones en pugna, más el sistema social).

- Un criterio subjetivo, elaborado a partir de la estimación que cada uno de los miembros de ambas delegaciones negociadoras tenga de su aceptabilidad para su respectivo grupo de referencia.

Ambos tipos de criterios "deben ser tenidos en cuenta, si se quiere comprender el proceso de la negociación, especificar los determinantes de dicho proceso y predecir sus resultados” (McGrath 1966:117).

\section{Conclusiones}

Desde una aproximación meramente cuantitativa, lo primero que se observa es que la mediación ha recibido menor atención que la negociación. Y es que parece haber consenso entre los distintos especialistas en considerar a la mediación como un caso particular de la negociación en la que el único factor de distinción sería la intervención de una tercera parte neutral junto con las partes en conflicto. Por eso, la mediación comparte con la negociación buena parte de los procesos, variables y dinámicas que se desatan cuando ambos tipos de técnicas de resolución pacífica de los conflictos se ponen en marcha. De ahí que la bibliografía sobre mediación se centre en buena medida en analizar el factor de distinción que la singulariza frente a la negociación: la presencia del mediador. Así, como hemos señalado más arriba, son las habilidades, prerrogativas y funciones del mediador las que ocupan el foco de atención de los autores que formulan sus análisis sobre la mediación.

Sin embargo, autores como Brunk (2000) se apartan de esta pauta al considerar que la mediación es una técnica de resolución pacífica de los conflictos que se emplea en dos situaciones: a) la resolución del conflicto no coercitiva y b) la gestión del conflicto no coercitiva. Mientras que la negociación funciona como técnica de resolución pacífica de los conflictos en los tres niveles (resolución, gestión y transformación) del conflicto. Por tanto, Brunk optaría por diferenciar la mediación de la negociación, hasta el punto de considerar que la introducción de la tercera parte neutral modifica a la negociación en el sentido de especializarla y convertirla en otra técnica, la mediación, más restringida y circunscrita a las dos situaciones ya mencionadas. Así pues la mediación no sirve para transformar el conflicto mientras que la negociación sirve para los tres niveles, tanto desde un punto de vista coercitivo como no coercitivo. ¿Por qué la mediación no es válida para encarar las causas estructurales que están en la raiz de un conflicto y la negociación si?. Una posible respuesta sería que la intervención de una tercera parte neutral se produce cuando las relaciones entre las partes contendientes son tan complicadas que no es posible que ambas se sienten en una mesa de diálogo y éste solo sea posible a través de la labor de intermediación del mediador. En una situación de esta naturaleza, es lógico concluir que dado el enconamiento de las posiciones de las partes en litigio únicamente nos podamos mover en las dimensiones de la gestión y la resolución del conflicto. Para avanzar hacia el nivel de la transformación del conflicto se requiere 
un mayor nivel de comunicación entre las partes. Y si esta mayor fluidez en la comunicación se logra ya no es necesaria la figura del mediador puesto que ambas partes pueden por si mismas comunicarse entre sí de forma directa. Es por ello que en el tercer nivel o enfoque distinguido por Brunk, la transformación del conflicto, la mediación no tiene sentido como técnica de resolución pacífica, dejando espacio para que se ensaye la negociación.

Ahora bien, sorprende que Brunk sitúe a la mediación del lado de las técnicas de resolución de conflictos "no coercitivas". En una primera aproximación parecería plausible considerar que la mediación al tener que contar necesariamente con el consenso de las partes enfrentadas tiene que producirse sin coerción. Sin embargo, en los desarrollos más recientes (Bercovitch 2009) ha aparecido un nuevo enfoque denominado "mediation with muscle" en el que ya no sería posible afirmar con rotundidad que todo proceso de mediación tiene que llevarse a cabo de modo no coercitivo. Un buen ejemplo de cómo es posible llevar a cabo un proceso de mediación en el que el mediador ejerce una influencia claramente coercitiva sobre las partes fueron los Acuerdos de Dayton que pusieron fin a la guerra en los Balcanes en la década de los noventa.

Dentro de los cuatro enfoques que en la bibliografía sobre mediación en el conflicto internacional distingue Bercovitch (1997), a saber, 1) Estudios prescriptivos 2) Estudios teóricos 3) Estudios basados en la Teoría de Juegos y 4) Estudios de caso, en este artículo nos hemos ocupado de realizar una revisión de las principales aportaciones que se han producido en el nivel 2, los estudios teóricos y más concretamente los estudios teóricos sobre la mediación como técnica de resolución de conflictos en la arena internacional.

\section{Referencias bibliográficas}

Bartunek J., Benton A. y Keys C. "Third Party Intervention and the Behavior of Group Representatives” en Journal of Conflict Resolution, 19 (3): 532-57.

Bercovitch J. (1997) "Mediation in International Conflict. An Overview of Theory, A Review of Practice" en Zartman W. and Rasmussen L. (eds.) Peacemaking in International Conflict. Methods \& Techniques, United States Institute of Peace Press, Washington.

Bercovitch J. y Rubin J. (1992) Mediation in International Relations, St. Martin's Press, New York.

Bercovitch J. y Sigmund Gartner, Scott (eds.) (2009) International Conflict Mediation: New Approaches and Findings, Routledge, New York.

Bingham G. (1985) Resolving Environmental Disputes, The Conservation Foundation, Washington, D.C. 
Blake R. And Mouton J. (1985) Solving Costly Organizational Conflicts, JosseyBass, S. Francisco.

Bouthoul G. (1970) Ganar la paz, Plaza y Janés, Barcelona.

Brunk C. (2000) "Shaping a vision: the nature of peace studies" en Fisk L. and Schellenberg J. (eds.) Patterns of conflict. Paths to peace, Broadview Press, Ontario.

Burton J. (1969) Conflict and Communication, MacMillan, London

Burton J. (1972) “The Resolution of Conflict” en International Studies Quaterly 16 (March): 5-29.

Burton J. (1984) Global Conflict, Wheatsheaf Books, Brighton, Sussex.

Carnevale P. (1986) "Strategic Choice in Mediation” en Negotiation Journal 2 (1), págs. 41-56.

Coser L. (1970) Nuevos aportes a la teoría del conflicto social, Amorrortu, Buenos Aires.

Doob L. (1971) Resolving Conflict in Africa, Yale University Press, New Haven.

Fisas V. (1987) Introducción al estudio de la paz y de los conflictos, Ed. Lerna, Barcelona.

Fisher R (1983) “Third-Party Consultation as a Method of Intergroup Conflict Resolution” en Journal of Conflict Resolution, 27(2): 301-44.

Fisher R. y Ury W. (1981) Getting to yes, Houghton Mifflin, Boston. (Exite traducción castellana: (1985) El arte de negociar sin ceder, Compañía editorial Continental, México).

Folberg J. and Taylor A. (1984) Mediation, Jossey-Bass, S. Francisco.

Harto de Vera F. (2005) Investigación para la paz y Resolución pacífica de conflictos, Ed. Tirant lo Blanch, Valencia.

Kellman H. (1992) "Informal Mediation by Scholar/Practicioner” en Bercovitch J. y Rubin J. (eds) Mediation in International Relations, St. Martin Press, New York.

Kolb D. (1983) "Who are organizational third parties and what do they do?" en Research on negotiation in organizations, Emerald Group Publising, Bradford.

McGrath J. (1966) "A social psychological approach to the study of negotiation” en Bowers R. Studies on behavior in organizations, University of Georgia Press, Athens.

Mitchell C. (1981) The Structure of International Conflict, Macmillan, London.

Moore C. (1986) The Mediation Process: Practical Strategies for Resolving Conflict, Jossey-Bass, San Francisco.

Ottt M. (1972) "Mediation as a Method of Conflict Resolution" en International Organization, 26(4): 595-618.

Raiffa H. (1982) The Art and Science of Negotiation, Harvard University Press, Cambridge, Mass.

Rubin J. (1980) "Experimental Research on Third-Party Intervention in Conflict" en Psychological Bulletin 87 (2): 379-91.

Rubin J. (ed.) (1981) Dynamics of Third-Party Intervention: Kissinger in the middle East, Praeger, New York. 
Schelling T. (1960) The Strategy of Conflict, Harvard University Press, Cambridge, Mass. Existe traducción española: (1964) La estrategia del conflicto, Tecnos, Madrid.

Singer L. (1990) Settling Disputes: Conflict Resolution in Bussines, Families and the Legal System, Westview, Boulder, Colorado.

Stein J. (1985) "Structure, Strategies and Tactics of Mediation" en Negotiation Journal 1(4): 331-47.

Stevens C. (1963) Strategy and Collective Bargaining Negotiations, McGraw Hill, New York.

Stulberg J. (1987) Taking Charge/Managing Conflict, D.C. Heath, Lexington, Mass. Touval S. and Zartman W. (eds.) (1985) The man in the Middle: International Mediation in Theory and Practice, Westview Press, Boulder, Colorado.

Wall J. (1981) "Mediation: An Analysis, Review and Proposed Research" en Journal of Conflict Resolution, 25 (1), pags. 157-80.

Walton R. (1969) Interpersonal Peacemaking:Confrontations and Third-Party Consultation, Addison-Wesley, Reading, Mass.

Young O. (1967) The Intermediaries: Third Parties in International Crises, Princeton University Press, Princeton. 